\title{
Januskinase-Inhibitoren sind Biologika mindestens ebenbürtig
}

Januskinase (JAK)-Inhibitoren haben sich in der Behandlung von Patienten mit rheumatoider Arthritis (RA), die auf konventionelle DMARDs ungenügend angesprochen haben, als Alternative zu Biologika etabliert.

In allen Phase-III-Studien wurde beim ACR-Ansprechen und den Remissionsraten nach DAS28-CRP die NichtUnterlegenheit von JAK-Inhibitoren gegenüber Adalimumab belegt, für Baricitinib und Upadacitinib sogar Überlegenheit, berichtete Professor Dr. Rubbert-Roth, Rheumatologin am Kantonsspital in St. Gallen. Mit Upadacitinib wurden in der 48-wöchigen SELECT Compare-Studie unabhängig vom Beurteilungskriterium (DAS28CRP, CDAl, SDAl, Bool'sche Remissionskriterien von $A C R$ und EULAR) ${ }^{1}$ höhere Remissionsraten als mit Adalimumab erzielt $[1,2]$.

\section{SELECT Choice-Studie: Überle- genheit von Upadacitinib vs. \\ Abatacept}

Ein ganz ähnliches Ergebnis wurde jetzt in SELECT Choice auch im Vergleich zu Abatacept erzielt. An der Studie nahmen mehr als 600 RA-Pati-

DAS: Disease Activity Score, CDAl: Clinical Disease Activity Index, SDAl: Simple Disease Activity Index enten teil, die mit unbefriedigendem Erfolg über mindestens drei Monate mit mindestens einem Biologikum behandelt worden waren oder die Therapie (unabhängig von der Dauer) nicht vertragen hatten.

Die Studienteilnehmer waren im Mittel 55 bis 56 Jahre alt, über $80 \%$ Frauen, erkrankt seit im Schnitt zwölf Jahren und zum Zeitpunkt der Randomisierung mit aktiver Erkrankung ( $\geq 6$ geschwollene und empfindliche Gelenke, DAS28 5,7 bis 5,9, hsCRP $\geq 3 \mathrm{mg} / \mathrm{l}$ ) [3]. Die Patienten wurden nun zusätzlich zur Basismedikation entweder mit Abatacept i.v. oder mit Upadacitinib (15 mg einmal täglich) behandelt.

Beim primären und sekundären Endpunkt - Veränderung des DAS28CRP in Woche $12 \mathrm{im}$ Vergleich zum Ausgangspunkt - konnte nicht nur die Nicht-Unterlegenheit, sondern auch die Überlegenheit gezeigt werden, berichtete Rubbert-Roth. $30 \%$ der Behandelten erreichten unter Upadacitinib eine Remission im Vergleich zu nur $13 \%$ unter Abatacept. Bei allen beur-

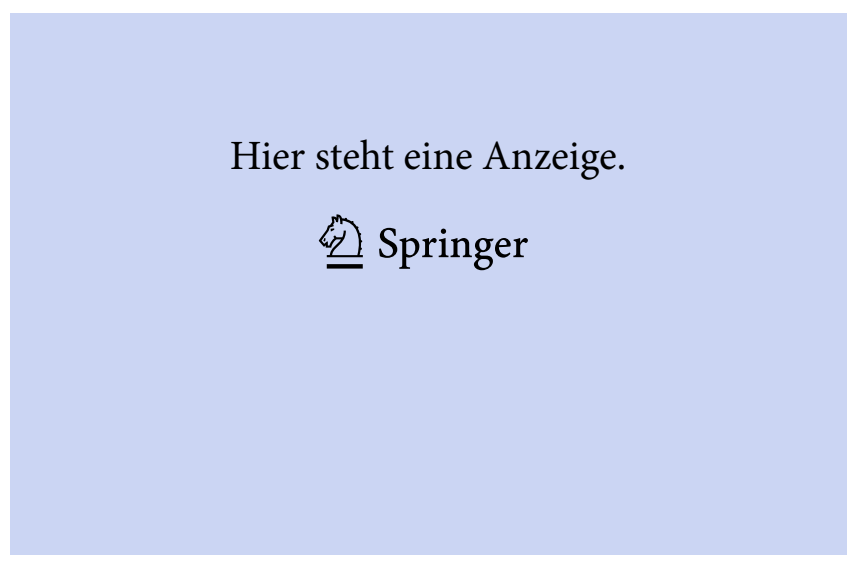

teilten Remissionsparametern wurden mit der Kombination von Upadacitinib plus Methotrexat höhere Raten als in der Vergleichsgruppe erreicht.

Die Raten unerwünschter Ereignisse waren in beiden Gruppen ähnlich (69\% Upadacitinib, 61,2\% Abatacept), etwas höher waren unter Upadacitinib die Raten ernsthafter (3,3\% vs. $1,6 \%)$ und schwerer Nebenwirkungen $(6,3 \%$ vs. $3,2 \%)$, darunter opportunistischer Infektionen (1,3\% vs. 0,3\%).

JAK-Inhibitoren scheinen in der RATherapie mindestens so effektiv wie Biologika zu sein, resümierte RubbertRoth. Welche Substanzklasse zuerst eingesetzt werde, sollte auch von der Patientenpräferenz abhängen.

\section{Roland Fath}

\section{Literatur \\ 1. Fleischmann RM et al (2019) Arthritis Rheu- matol 71:788-1800 \\ 2. Fleischmann RM et al (2019) Arthritis Rheu- matol 78:1454-1462 \\ 3. Rubbert-Roth A et al (2020) EULAR, Abstract SAT0151}

Hinweis des Verlags. Der Verlag bleibt in Hinblick auf geografische Zuordnungen und Gebietsbezeichnungen in veröffentlichten Karten und Institutsadressen neutral.

rheuma plus $2020 \cdot 19: 177$

https://doi.org/10.1007/s12688-02000368-w

๑) Springer-Verlag GmbH Austria, ein Teil von Springer Nature 2020
Quelle: Meet the Expert: "How do JAK inhibitors compare to established RA therapies? A review of active comparator studies", im Rahmen des E-Kongresses EULAR 2020, 5. Juni 2020 